\title{
Special issue forward: the 2018 Mixed Integer Programming workshop
}

\author{
Daniel Bienstock ${ }^{1} \cdot$ Giacomo Nannicini $^{2}$
}

Published online: 20 May 2020

() Springer-Verlag GmbH Germany, part of Springer Nature and Mathematical Optimization Society 2020

The 2018 Mixed Integer Programming workshop took place at Clemson University (Greenville, South Carolina) June 18-21. The goal of the MIP workshop series is to discuss recent research in Mixed Integer Programming, and the 2018 edition consisted of a single track of 20 invited talks, together with a poster session with 26 posters. The six papers published in this special volume are related to talks presented at the workshop. All the papers underwent a rigorous peer-review process; we are very grateful to the anonymous referees, and to the guest editors who handled the submissions for this special issue (Claudia D'Ambrosio, Marcos Goycoolea, Oktay Gunluk, Jim Luedtke, Michele Monaci, Jean-Philippe Richard). We are also grateful to the local organization committee (Akshay Gupte, Matthew Saltzman, Cole Smith) for making this workshop possible.

On behalf of the program committee (Philipp Christophel, Simge Küçükyavuz, Ruth Misener, Giacomo Nannicini, Alejandro Toriello).

Daniel Bienstock and Giacomo Nannicini, New York, May 2020.

Publisher's Note Springer Nature remains neutral with regard to jurisdictional claims in published maps and institutional affiliations.

Giacomo Nannicini nannicini@us.ibm.com

Daniel Bienstock dano@columbia.edu

1 Columbia University, New York, NY, USA

2 IBM T.J. Watson Research Center, Yorktown Heights, NY, USA 